\title{
Efficacy and safety of Reduning injection in the treatment of COVID-19: a randomized, multicenter clinical study
}

\author{
Xiaolong Xu ${ }^{1}$, Junhua Zhang ${ }^{2}$, Wenke Zheng ${ }^{2}$, Zifeng Yang ${ }^{3}$, Xuegong Zhao ${ }^{4}$, Changfeng Wang ${ }^{5}$, \\ Hongzheng Su${ }^{6}, \mathrm{Li} \mathrm{Zhao}^{7}$, Leyang Xue ${ }^{8}$, Fen Hu${ }^{9}$, Xiaohong Xu ${ }^{10}$, Minyong Wen ${ }^{11}$, Jiong Liao ${ }^{12}$, \\ Zhaohai Zeng ${ }^{13}$, Liping Wang ${ }^{14}$, Jiaqing Zeng ${ }^{15}$, Yuhong Guo ${ }^{1}$, Bo Li ${ }^{1}$, Qingquan Liu ${ }^{1}$
}

${ }^{1}$ Beijing Hospital of Traditional Chinese Medicine, Capital Medical University, Beijing Institute of Traditional Chinese Medicine, Beijing, China; ${ }^{2}$ Institute of Traditional Chinese Medicine, Tianjin University of Traditional Chinese Medicine, Tianjin, China; ${ }^{3}$ Guangzhou Institute of Respiratory Diseases, Guangzhou, China; ${ }^{4}$ The Third Hospital of Yichang, Yichang, China; ${ }^{5}$ Huanggang Central Hospital, Huanggang, China; ${ }^{6}$ People's Hospital of Zaoyang, Zaoyang, China; ${ }^{7}$ The Fourth People's Hospital of Lianyungang, Lianyungang, China; ${ }^{8}$ Huai'an Fourth People's Hospital, Huai'an, China; 'The First People's Hospital of Jiangxia District of Wuhan City, Wuhan, China; ${ }^{10}$ People's Hospital of Hanchuan, Hanchuan, China; ${ }^{11}$ The First Affiliated Hospital of Guangzhou University of Chinese Medicine, Guangzhou, China; ${ }^{12}$ People's Hospital of Laibin, Laibin, China; ${ }^{13}$ People's Hospital of Guangshan County, Xinyang, China; ${ }^{14}$ The Affiliated Hospital of Xuzhou Medical University, Xuzhou, China; ${ }^{15}$ The First People's Hospital of Laohekou, Laohekou, China

Contributions: (I) Conception and design: Q Liu, J Zhang; (II) Administrative support: Q Liu; (III) Provision of study materials or patients: W Zheng, Z Yang, X Zhao, C Wang, H Su, L Zhao, L Xue, F Hu, X Xu, M Wen, J Liao, Z Zeng, L Wang, J Zeng; (IV) Collection and assembly of data: W Zheng, Z Yang, X Zhao, C Wang, H Su, L Zhao, L Xue, F Hu, X Xu, M Wen, J Liao, Z Zeng, L Wang, J Zeng; (V) Data analysis and interpretation: X Xu, Y Guo, B Li; (VI) Manuscript writing: All authors; (VII) Final approval of manuscript: All authors.

Correspondence to: Qingquan Liu. Beijing Hospital of Traditional Chinese Medicine, Affiliated with Capital Medical University, 23 Meishuguan Backstreet, Dongcheng District, Beijing 100010, China. Email: liuqingquan2003@126.com.

Backgroundk Reduning injection is a traditional Chinese medicine (TCM) with known efficacy against a variety of viral infections, but there is no data about its efficacy against coronavirus disease 2019 (COVID-19).

Methods: To explore the efficacy and safety of Reduning injection in the treatment of COVID-19, a randomized, open-labeled, multicenter, controlled trial was conducted from 12 general hospitals between 2020.02.06 and 2020.03.23. Patients with COVID-19 who met the diagnostic criteria of the "Diagnosis and Treatment Program for Novel Coronavirus Infection Pneumonia (Trial Fifth Edition)". Patients were randomized to routine treatment with or without Reduning injection (20 mL/day for 14 days) (ChiCTR2000029589). The primary endpoint was the rate of achieving clinical symptom recovery on day 14 of treatment.

Results: There were 77 and 80 participants in the Reduning and control groups. The symptom resolution rate at 14 days was higher in the Reduning injection than in controls [full-analysis set (FAS): $84.4 \%$ vs. $60.0 \%, \mathrm{P}=0.0004]$. Compared with controls, the Reduning group showed shorter median time to resolution of the clinical symptoms (143 vs. $313.5 \mathrm{~h}, \mathrm{P}<0.001)$, shorter to nucleic acid test turning negative (146.5 vs. $255.5 \mathrm{~h}, \mathrm{P}<0.001)$, shorter hospital stay (14.1 vs. 18.1 days, $\mathrm{P}<0.001)$, and shorter time to defervescence (29 vs. $71 \mathrm{~h}, \mathrm{P}<0.001)$. There was no difference in AEs (3.9\% vs. 8.8\%, $\mathrm{P}=0.383$ ).

Conclusions: This preliminary trial suggests that Reduning injection might be effective and safe in patients with symptomatic COVID-19.

Keywords: Coronavirus disease 2019 (COVID-19); randomized controlled trial; traditional Chinese medicine (TCM); efficacy and safety 
Submitted Oct 27, 2020. Accepted for publication Mar 25, 2021.

doi: 10.21037/apm-20-2121

View this article at: http://dx.doi.org/10.21037/apm-20-2121

\section{Introduction}

Coronavirus disease 2019 (COVID-19) is a global pandemic of acute respiratory disease caused by a novel coronavirus (SARS-CoV-2) (1). SARS-CoV- 2 is a member of the $\beta$-genus coronaviruses and is closely related to SARS-CoV (2). The first cases were reported in Wuhan in December 2019. On June 30, 2020, the World Health Organization (WHO) reported that nearly all countries were affected, with a total of 10,185,374 cases and 503,862 deaths (WHO data). The common signs of COVID-19 include fever, cough, and shortness of breath $(1,3,4)$. Severe cases can progress to severe acute respiratory syndrome (SARS) that will require oxygen and respiratory support, and that may progress to death (3-5).

There is no specific antiviral treatment for COVID-19, but supportive care performed on a case-by-case basis may help relieve symptoms and should include support of vital organ functions in severe cases (6-8). Supportive therapies are currently the keystone to COVID-19 treatment (6-8), and the development of vaccines and new drugs takes time. Traditional Chinese medicine (TCM) has a long history in the treatment of viral respiratory diseases such as influenza (9). Recently, some TCM compounds were explored for efficacy against COVID-19 (10). Recent studies examined the effect of the Lianhuaqingwen capsule and showed that it could shorten the course of the disease and improve the patients' condition (11-13).

Reduning injection is a TCM that possesses some efficacy against viral diseases such as $\mathrm{H} 1 \mathrm{~N} 1$ influenza A, hand-foot-mouth disease, and pneumonia (14-17). Reduning injection is composed of three herbs (Artemisia annua, Lonicera japonica Thunb, and Gardenia jasminoides Ellis), and its main functions are "clearing heat, relieving wind and detoxifying". The indications for treatment include hyperpyrexia, mild nausea and wind cold, headache, pain, cough, and yellow phlegm, as well as upper respiratory tract infections with the above symptoms (14-17). In Reduning, Artemisia apiacea is the major ingredient, with effects of clearing heat and cooling blood, and penetrating and dispersing muscle surface. Lonicera japonica Tbunb is a supporting drug, with effects of clearing heat and detoxifying, evacuating wind and heat to help Artemisia apiacea clearing heat, penetrating, and dispersing. Fructus gardenia is an assistant drug, with effects of clearing heat and removing dampness, cooling blood, and detoxifying. The combination of those drugs has effects of clearing heat, evacuating wind, and detoxifying. Modern pharmacological studies showed that Reduning injection has effects such as antiviral, antipyretic, and anti-inflammatory storm $(14,15)$. Reduning injection was included in the "Diagnosis and Treatmnt Program for Influenza a H1N1 Influenza" by the Chinese Ministry of Health in 2010, the "Chinese Medicine Guidelines for diagnosis and treatment of viral pneumonia in children" in 2011, the "Hospital Infection Prevention and Control Technical Guidelines for Middle East Respiratory Syndrome" in 2015 and a total of 12 guidelines.

Nevertheless, despite its known efficacy for a variety of viral infections, there is no data about the efficacy of Reduning injection against COVID-19. Therefore, the aim of the present trial was to explore the efficacy and safety of Reduning injection in the treatment of COVID-19. The results might suggest a novel drug in the armamentarium against COVID-19. We present the following article in accordance with the CONSORT reporting checklist (available at http://dx.doi.org/10.21037/apm-20-2121).

\section{Methods}

\section{Study design and participants}

This was a randomized, open-labeled, multicenter, controlled trial of patients with COVID-19 who met the diagnostic criteria of the "Diagnosis and Treatment Program for Novel Coronavirus Infection Pneumonia (Trial Fifth Edition)" (18) (Appendix 1) were included from 12 general hospitals between 2020.02.06 and 2020.03.23. The study was approved by the Ethics Committee of Tianjin University of Traditional Chinese Medicine (No. RDN2019-nCoV-1.1). The study followed the GCP guidelines and the Declaration of Helsinki (as revised in 2013). Written informed consent was obtained from the patients or legal representatives. The study was registered in Chinese Clinical Trial Registry (ChiCTR2000029589).

The inclusion criteria were: (I) diagnosed with COVID-19; (II) one or more symptoms among fever, cough, 
and fatigue at enrollment; (III) the time from onset to diagnosis time was not more than $48 \mathrm{~h}$; and (IV) $\geq 18$ years of age. The exclusion criteria were: (I) immunodeficiency, immunosuppressive drugs, or glucocorticoids within the past 3 months; (II) preparing for pregnancy, pregnant women, or lactating women; (III) allergies to more than two types of drugs or foods or allergies to ingredients of the trial drug; (IV) psychosis or those without self-knowledge ability; (V) estimated survival time less than $48 \mathrm{~h}$ from the start of screening; (VI) tracheal intubation was already done before screening; or (VII) those who were judged by the investigators to be not suitable to participate in the trial.

\section{Randomization and grouping}

A simple randomization method was used to randomly group the participants, based on the last digit of their ID card. Those with an even number entered the Reduning group, and those with an odd number entered the control group. The participants in the Reduning group received routine treatment + Reduning injection, while the control group received routine treatment. This was an open-label trial, and there was no blinding.

\section{Reduning injection}

Reduning injection is a Class II new TCM. It obtained a new drug certificate and production approval from the State Food and Drug Administration on May 10, 2005 (National Pharmaceutical Standard \#Z20050217). Reduning injection is composed of three TCM herbs: Artemisia annua, Lonicera japonica Thunb, and Gardenia jasminoides Ellis. It has antiviral, anti-inflammatory, antipyretic, antibacterial, and immunomodulatory effects.

\section{Treatment methods}

For routine management, all treatments complied with the "Diagnosis and Treatment Program for Novel Coronavirus Infection Pneumonia (Trial Fifth Edition)", including supportive treatment (oxygen), antiviral treatment, and symptomatic treatment. For Reduning treatment, $20 \mathrm{~mL}$ of Reduning injection was diluted with $250 \mathrm{~mL}$ of saline, and then intravenously infused once a day for 2 weeks.

\section{Endpoints}

The primary endpoint was the rate of achieving clinical symptom recovery on day 14 of treatment. The standard of clinical symptom recovery was the disappearance of the main symptoms, such as fever, cough, and fatigue. The secondary endpoints included the proportion of patients who achieved clinical symptom recovery on days 7 and 10, the time for clinical recovery of the patients, the time for nucleic acid turning negative (defined as two consecutive negative nucleic acid tests, at least 1 day apart for respiratory tract pathogens), time for the first negative test result, hospitalization duration [the criteria for release from quarantine and discharge were according to the "Diagnosis and Treatment Program for Novel Coronavirus Infection Pneumonia (Trial Fifth Edition)"], and time for defervescence (the time required to reach $<37.4{ }^{\circ} \mathrm{C}$ after starting medication and maintained for at least $24 \mathrm{~h}$ or more).

\section{Data collection and definition}

The sex, age, height, weight, admission temperature, CURB-65 score (19), disease grading [mild, moderate, severe, and critical according to the "Diagnosis and Treatment Program for Novel Coronavirus Infection Pneumonia (Trial Fifth Edition)"], clinical symptoms (fever, fatigue, cough, wheezes, and sputum), and medication (antiviral drugs, antibacterial drugs, and hormones) were collected.

\section{Safety}

All adverse events (AEs), defined as any adverse medical events that occurred from the beginning of the patient's enrollment to the end of the trial, regardless of whether there was a causal relationship with the trial drug, that occurred in the trial were recorded.

\section{Statistical analysis}

As this is a preliminary trial and no previous data were available, a power analysis was not performed when designing the trial, but a post hoc power analysis was performed. The full-analysis set (FAS) included all enrolled patients who had received the test drug at least once and had at least one assessment record. The missing effective indicators were estimated using the last observation carried forward (LOCF) method. The FAS population was used for the analysis of the primary and secondary efficacy indicators. The per-protocol set (PPS) included all participants that complied with the trial protocol, had good compliance, did 


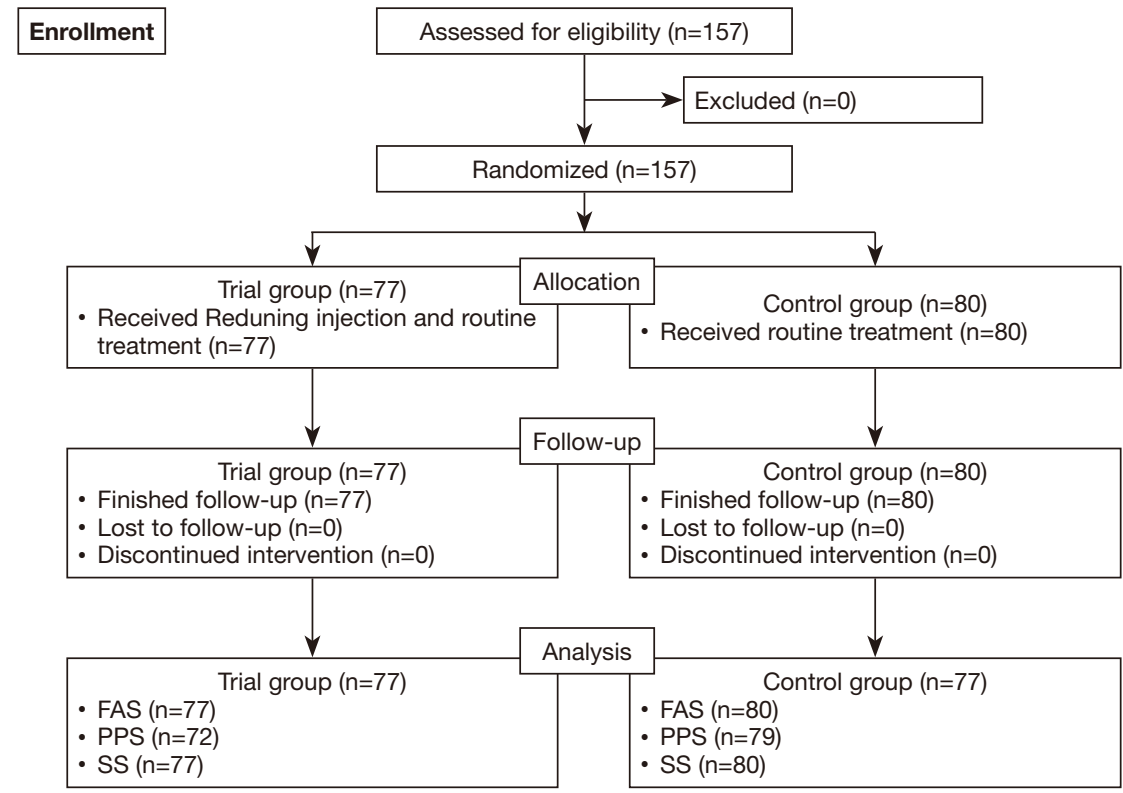

Figure 1 Participant flowchart. FAS, full-analysis set; PPS, per-protocol set; SS, safety analysis set.

not use forbidden drugs during the trial, and completed the contents specified in the case report form (CRF). The PPS population was used for the analysis of the primary and secondary efficacy indicators. The safety analysis set (SS) referred to the participants that received at least one study treatment and had baseline data of safety and at least one visit for safety. The SS was the main population for the safety evaluation in this study.

All analyses were performed using SAS 9.4 (order number: 9C1XJD, SAS Institute, Cary, NY, USA). After verifying the distribution of the continuous variables using the Kolmogorov-Smirnov test, the data were presented as means \pm standard deviations and compared using Student's $t$-test. The categorical variables were expressed as numbers (percentage) and analyzed using the chi-square test or Fisher's exact test. Kaplan-Meier curves were drawn for time for clinical symptom recovery, time for nucleic acid turning negative, length of hospital stay, and time for defervescence; they were analyzed using the log-rank test. All tests two-tailed (except the chi-square test) and $\mathrm{P}$ values $<0.05$ were considered statistically significant.

\section{Results}

\section{Characteristics of the patients}

Figure 1 presents the patient flowchart. A total of 157 patients confirmed with COVID-19 from 12 general hospitals were enrolled and randomized between 2020.02.06 and 2020.03.23. Among them, there were 77 and 80 participants in the Reduning and control groups, respectively, with 77 and 80 in the FAS and SS, and 72 and 79 in the PPS. All 157 participants completed the study. Table 1 shows that the two groups were similar regarding age, sex, temperature at onset, CURB-65 score, symptoms, and combined medication (all $\mathrm{P}>0.05$ ).

\section{Efficacy}

Table 2 presents the efficacy results. The symptom resolution rate at 14 days was higher in the Reduning injection than in the control group (FAS: $84.4 \%$ vs. $60.0 \%$, $\mathrm{P}=0.0004$; PPS: $86.1 \%$ vs. $59.5 \%, \mathrm{P}=0.0002)$. Similar results were also observed earlier in the treatment course, i.e., at 7 days (FAS: $81.8 \%$ vs. $23.8 \%, \mathrm{P}<0.0001$; PPS: $81.9 \%$ vs. $22.8 \%, \mathrm{P}<0.0001)$ and 10 days (FAS: $84.4 \%$ vs. $48.9 \%$, $\mathrm{P}<0.0001$; PPS: $84.7 \%$ vs. $48.1 \%, \mathrm{P}<0.0001)$. In the $\mathrm{FAS}$, compared with the control group, the Reduning group had a shorter median time to resolution of the clinical symptoms (143 vs. $313.5 \mathrm{~h}, \mathrm{P}<0.001$ ) (Figure 2), shorter to nucleic acid test turning negative (146.5 vs. $255.5 \mathrm{~h}, \mathrm{P}<0.001)$ (Figure 3), shorter hospital stay (14.1 vs. 18.1 days, $\mathrm{P}<0.001)$ (Figure 4), and shorter time to defervescence $(29$ vs. $71 \mathrm{~h}, \mathrm{P}<0.001)$ 
Table 1 Baseline characteristics

\begin{tabular}{|c|c|c|c|c|}
\hline Indicators & All $(n=157)$ & Reduning group $(n=77)$ & Control group $(n=80)$ & $\mathrm{P}$ \\
\hline$>65$ years old, $\mathrm{n}(\%)$ & $26(16.6)$ & $12(15.6)$ & $14(17.5)$ & 0.7469 \\
\hline Male, n (\%) & $87(55.4)$ & $43(55.8)$ & $44(55.0)$ & 0.9153 \\
\hline Temperature at onset $\left({ }^{\circ} \mathrm{C}\right)$ & $37.5 \pm 0.7$ & $37.5 \pm 0.8$ & $37.5 \pm 0.7$ & 0.7462 \\
\hline Mild & $5(3.2)$ & $4(5.2)$ & $1(1.3)$ & \\
\hline Moderate & $132(84.1)$ & $67(87.0)$ & $65(81.3)$ & \\
\hline Severe & $20(12.7)$ & $6(7.8)$ & $14(17.5)$ & \\
\hline CURB-65 >3, n (\%) & $133(84.7)$ & $65(84.4)$ & $68(85.0)$ & 0.9190 \\
\hline Fatigue & $69(44.0)$ & $38(49.4)$ & $31(38.8)$ & 0.1809 \\
\hline Cough & $115(73.3)$ & $60(77.9)$ & $55(68.8)$ & 0.1943 \\
\hline Wheeze & $8(5.1)$ & $5(6.5)$ & $3(3.8)$ & 0.4345 \\
\hline Sputum & $14(8.9)$ & $8(10.4)$ & $6(7.5)$ & 0.5254 \\
\hline \multicolumn{5}{|c|}{ Combined medication, $\mathrm{n}(\%)$} \\
\hline Antiviral agents & $155(98.7)$ & 75 (97.4) & $80(100.0)$ & 0.2389 \\
\hline Antibacterial agents & $116(73.9)$ & $61(79.2)$ & $55(68.8)$ & 0.1496 \\
\hline Hormones & 53 (33.8) & $20(26.0)$ & 33 (41.3) & 0.0628 \\
\hline
\end{tabular}

The data of patients, including sex, age, height, weight, admission temperature, and CURB-65 score were listed. CURB-65, Confusion, Urea, Respiratory rate and Age 65.

Table 2 Cure rates of symptoms on days 7,10 , and 14

\begin{tabular}{|c|c|c|c|c|c|c|}
\hline Time & \multicolumn{3}{|c|}{ FAS } & \multicolumn{3}{|c|}{ PPS } \\
\hline $7^{\text {th }}$ day, n (\%) & $63(81.8)$ & $19(23.8)$ & $<0.0001$ & $59(81.9)$ & $18(22.8)$ & $<0.0001$ \\
\hline $10^{\text {th }}$ day, $\mathrm{n}(\%)$ & 65 (84.4) & $39(48.8)$ & $<0.0001$ & $61(84.7)$ & 38 (48.1) & $<0.0001$ \\
\hline $14^{\text {th }}$ day, n (\%) & 65 (84.4) & $48(60.0)$ & 0.0004 & $62(86.1)$ & 47 (59.5) & 0.0002 \\
\hline
\end{tabular}

FAS, full-analysis set; PPS, per-protocol set.

(Figure S1). Similar results were observed in the PPS. Taken together, Reduning led to better resolution rate at 14 days of treatment, which involved a shorter time to symptom resolution, shorter time to negative nucleic acid test, and faster resolution of fever, which contributed together to shorter hospitalization.

\section{Safety}

Table 3 presents the safety data. There was no difference in the occurrence of $\mathrm{AEs}$ between the two groups (Reduning: $3.9 \%$ vs. controls: $8.8 \%, \mathrm{P}=0.383)$. There were three deaths in the control group and none in the Reduning group. 

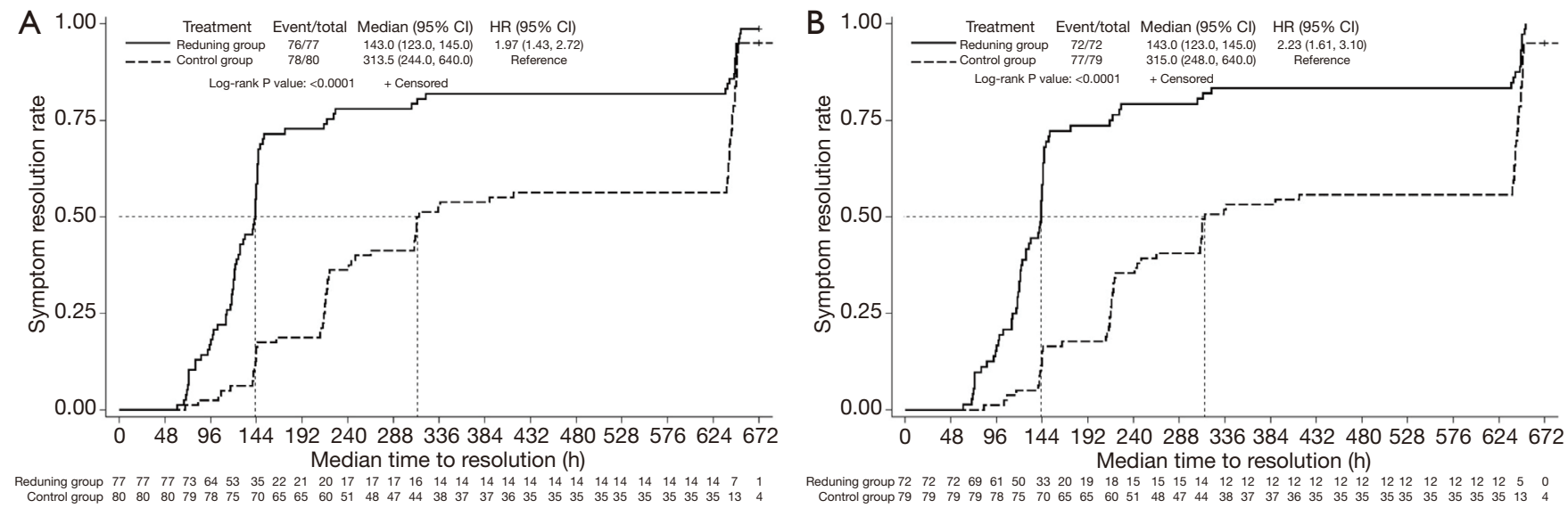

Figure 2 Time for the resolution of the clinical symptoms. (A) Full-analysis set (FAS); (B) per-protocol set (PPS). The blue line represents the control group.
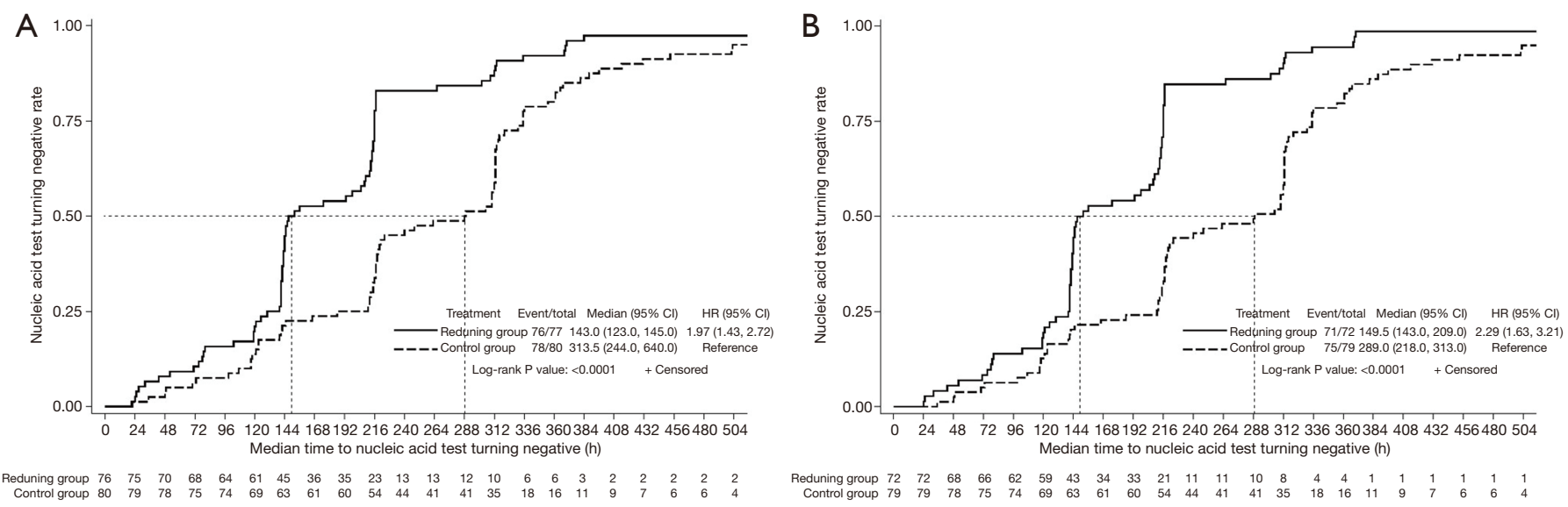

Figure 3 Time to nucleic acids turning negative. (A) Full-analysis set (FAS); (B) per-protocol set (PPS).

The AEs in the Reduning group were nausea $(2 / 77,2.6 \%)$, diarrhea $(1 / 77,1.3 \%)$, and stomach discomfort $(1.77,1.3 \%)$.

\section{Post-hoc power analysis}

Based on the results for the primary endpoint in the FAS and considering $\alpha=0.05$, this trial had a power of $90-95 \%$, as calculated using StatMate 2.0 (GraphPad Software Inc., San Diego, CA, USA). Therefore, the post hoc power analysis suggests that the study was adequately powered to detect differences in the symptom resolution rate at 14 days.

\section{Survival}

Table S1 shows that the difference in the 28-day survival of the participants was not statistically significant (FAS: $100 \%$ vs. $96.3 \%, \mathrm{P}=0.246$; PPS: $100 \%$ vs. $96.2 \%, \mathrm{P}=0.247)$.

\section{Discussion}

Reduning injection is a TCM with known efficacy against a variety of viral infections such as H1N1 influenza A, hand-foot-mouth disease, and acute bronchitis, but there 

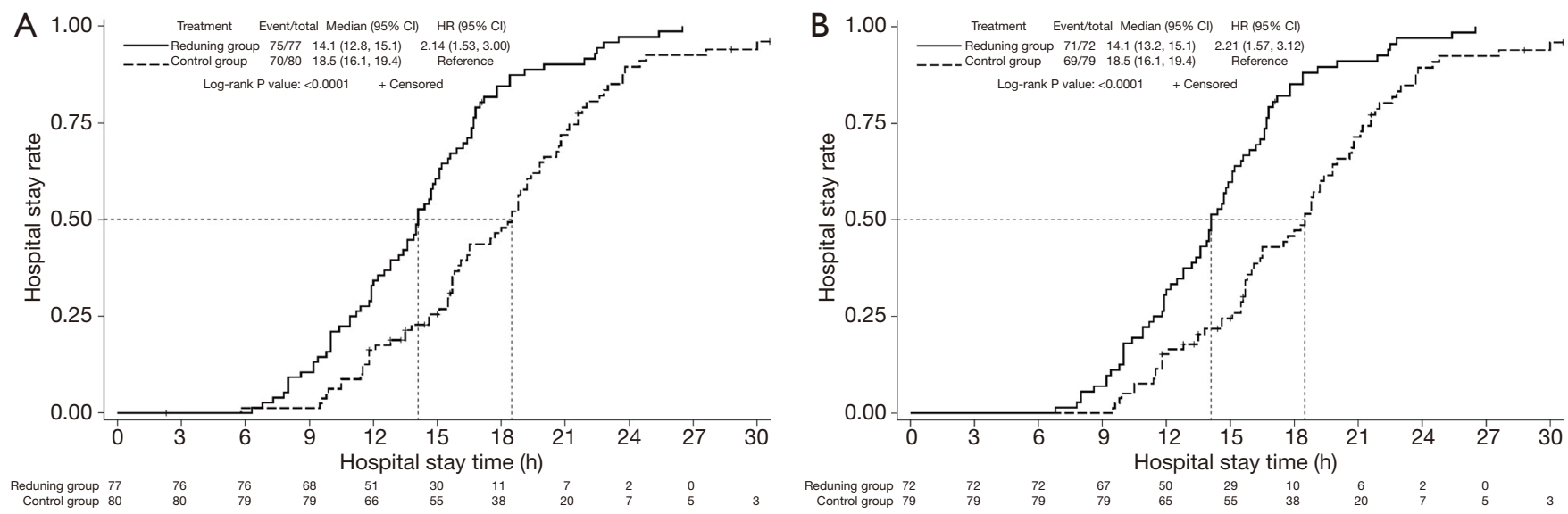

Figure 4 Length of hospital stay. (A) Full-analysis set (FAS); (B) per-protocol set (PPS).

Table 3 AEs

\begin{tabular}{lcc}
\hline Events & Reduning group $(\mathrm{n}=77)$ & Control group $(\mathrm{n}=80)$ \\
\hline AEs, $\mathrm{n}(\%)$ & $3(3.9)$ & $7(8.8)$ \\
Death, $\mathrm{n}(\%)$ & 0 & $3(3.8)$ \\
Abnormal liver function, $\mathrm{n}(\%)$ & 0 & $2(2.5)$ \\
Nausea, $\mathrm{n}(\%)$ & $2(2.6)$ & $1(1.3)$ \\
Diarrhea, $\mathrm{n}(\%)$ & $1(1.3)$ & 0 \\
Bitter taste, $\mathrm{n}(\%)$ & 0 & $1(1.3)$ \\
Stomach discomfort, $\mathrm{n}(\%)$ & $1(1.3)$ & 0 \\
Poor appetite, $\mathrm{n}(\%)$ & 0 & $1(1.3)$ \\
\hline
\end{tabular}

Data of AEs were recorded. AE, adverse event.

is no data about its efficacy against COVID-19. Therefore, this randomized, open-labeled, multicenter, controlled trial aimed to explore the efficacy and safety of Reduning injection in the treatment of COVID-19. This preliminary trial suggests that Reduning injection might be effective and safe in patients with symptomatic COVID-19.

The absence of a vaccine and effective drugs against SARS-CoV-2, and the lack of time to develop an entirely novel drug class, limit the current treatments of COVID-19 to supportive treatments $(1,20)$. Different drugs were tried, including HIV protease inhibitors (21-23), nucleoside analogs (24), chloroquine (24), and corticosteroids (25-27), but with uncertain efficacy and sometimes significant side effects (28).

A number of TCM preparations are known to possess efficacy against viral infections, including SARS-CoV (28).
Some TCM compounds are known to possess direct effects on viral proteins (29). TCM was extensively used for the management of SARS-CoV and, given the similarity between SARS-CoV and SARS-CoV-2, some evidence from SARS-CoV could be used (28). A number of clinical trials are underway for the efficacy and safety of TCM against COVID-19, but the present trial is the only one on Reduning. Published data showed that the Lianhuaqingwen capsule and showed that it could shorten the course of the disease and improve the patients' condition $(9,11,12)$. In the same manner, Reduning had a marked efficacy in terms of symptom resolution rates at 7, 10, and 14 days, time to resolution of symptoms, time to defervescence, hospitalization, and time to a negative nucleic acid test. Nevertheless, whether a TCM is superior to another against COVID-19 will have to be evaluated with head-to-head 
comparisons within the same trial.

Reduning injection is composed of three herbs: Artemisia annua, Lonicera japonica Thunb, and Gardenia jasminoides Ellis. Artemisinin and artesunate have known anti-malaria and antiviral properties (30), including against hepatitis B virus, bovine viral diarrhea virus, EpsteinBarr virus, and SARS-CoV (31,32). Lonicera japonica Thunb contains microRNA2911, which directly targets the influenza A virus (33). Iridoid glycoside extracts from Gardenia jasminoides Ellis also directly inhibit the influenza A virus (34). Therefore, the three components of Reduning injection possess proven antiviral properties, but which compounds are effective against COVID-19 will require additional study.

The present study showed that the occurrence of AEs was similar in the two groups. Of note, the only three deaths were observed in the control group, but it is unknown whether additional deaths were prevented by Reduning injection. Of note, Reduning injection is known to have allergenic compounds (35). Although no allergic reactions or anaphylactic shocks were observed in the present trial, it remains a possibility that they have to be considered when giving the treatment.

This study has limitations. This study was an exploratory and open-labeled trial, and the sample size was not calculated before conducing it. Only a small number of patients were included, which did not allow stratification based on disease severity. A variety of supportive drugs had to be given based on the guidelines (18), and their influence on the results could not be controlled and might constitute a bias. Because COVID-19 is an international emergency and treatment was the first priority, the randomization method was not standardized, and only a semi-random method was used.

In conclusion, this preliminary trial suggests that Reduning injection might be effective and safe in patients with symptomatic COVID-19. Additional studies are necessary to confirm the results.

\section{Acknowledgments}

The authors acknowledge the help of academician Zhang Boli from Chinese Academy of Engineering, Dr. Zhao Binjiang from Jiangsu Kanion pharmaceutical, and Dr. Chenqiong from Shanghai Medsci.

Funding: This study was funded by the National Natural Science Foundation of China (No. 62041701), the Special Project of Ministry of Science and Technology of China
(No. 2020YFC0841600), and the National Major Scientific and Technological Project (No. 2017ZX10305501).

\section{Footnote}

Reporting Checklist: The authors have completed the CONSORT reporting checklist. Available at http://dx.doi. org/10.21037/apm-20-2121

Data Sharing Statement: Available at http://dx.doi. org/10.21037/apm-20-2121

Conflicts of Interest: All authors have completed the ICMJE uniform disclosure form (available at http://dx.doi. org/10.21037/apm-20-2121). The authors have no conflicts of interest to declare.

Ethical Statement: The authors are accountable for all aspects of the work in ensuring that questions related to the accuracy or integrity of any part of the work are appropriately investigated and resolved. The study was approved by the Ethics Committee of Tianjin University of Traditional Chinese Medicine (No. RDN-2019-nCoV-1.1). The study followed the GCP guidelines and the Declaration of Helsinki (as revised in 2013). Written informed consent was obtained from the patients or legal representatives.

Open Access Statement: This is an Open Access article distributed in accordance with the Creative Commons Attribution-NonCommercial-NoDerivs 4.0 International License (CC BY-NC-ND 4.0), which permits the noncommercial replication and distribution of the article with the strict proviso that no changes or edits are made and the original work is properly cited (including links to both the formal publication through the relevant DOI and the license). See: https://creativecommons.org/licenses/by-nc-nd/4.0/.

\section{References}

1. Wiersinga WJ, Rhodes A, Cheng AC, et al. Pathophysiology, transmission, diagnosis, and treatment of coronavirus disease 2019 (COVID-19): a review. JAMA 2020;324:782-93.

2. Coronaviridae Study Group of the International Committee on Taxonomy of Viruses. The species Severe acute respiratory syndrome-related coronavirus: classifying 2019-nCoV and naming it SARS-CoV-2. Nat Microbiol 2020;5:536-44. 
3. Chen T, Wu D, Chen H, et al. Clinical characteristics of 113 deceased patients with coronavirus disease 2019: retrospective study. BMJ 2020;368:m1091.

4. Lewnard JA, Liu VX, Jackson ML, et al. Incidence, clinical outcomes, and transmission dynamics of severe coronavirus disease 2019 in California and Washington: prospective cohort study. BMJ 2020;369:m1923.

5. Richardson S, Hirsch JS, Narasimhan M, et al. Presenting characteristics, comorbidities, and outcomes among 5700 patients hospitalized with COVID-19 in the New York City area. JAMA 2020;323:2052-9.

6. Sharma O, Sultan AA, Ding H, et al. A review of the progress and challenges of developing a vaccine for COVID-19. Front Immunol 2020;11:585354.

7. Poland GA, Ovsyannikova IG, Kennedy RB. SARS-CoV-2 immunity: review and applications to phase 3 vaccine candidates. Lancet 2020;396:1595-606.

8. Odusanya OO, Odugbemi BA, Odugbemi TO, et al. COVID-19: a review of the effectiveness of nonpharmacological interventions. Niger Postgrad Med J 2020;27:261-7.

9. Duan ZP, Jia ZH, Zhang J, et al. Natural herbal medicine Lianhuaqingwen capsule anti-influenza A (H1N1) trial: a randomized, double blind, positive controlled clinical trial. Chin Med J (Engl) 2011;124:2925-33.

10. Wang M, Cao R, Zhang L, et al. Remdesivir and chloroquine effectively inhibit the recently emerged novel coronavirus $(2019-\mathrm{nCoV})$ in vitro. Cell Res 2020;30:269-71.

11. Hu K, Guan WJ, Bi Y, et al. Efficacy and safety of Lianhuaqingwen capsules, a repurposed Chinese herb, in patients with coronavirus disease 2019: a multicenter, prospective, randomized controlled trial. Phytomedicine 2021;85:153242.

12. Li RF, Hou YL, Huang JC, et al. Lianhuaqingwen exerts anti-viral and anti-inflammatory activity against novel coronavirus (SARS-CoV-2). Pharmacol Res 2020;156:104761.

13. Wang C, Hou J, Yan S, et al. Chinese herbal medicine therapy for coronary heart disease complicated with anxiety: a systematic review of randomized controlled trials. J Tradit Chin Med 2020;40:1-16.

14. Xie F, Xie M, Yang Y, et al. Assessing the anti-inflammatory mechanism of Reduning injection by network pharmacology. Biomed Res Int 2020;2020:6134098.

15. Cao C, Zhen Z, Kuang S, et al. Reduning injection combined with western medicine for pneumonia: a protocol for systematic review and meta-analysis. Medicine
(Baltimore) 2020;99:e22757.

16. Zhang G, Zhao J, He L, et al. Reduning injection for fever, rash, and ulcers in children with mild hand, foot, and mouth disease: a randomized controlled clinical study. J Tradit Chin Med 2013;33:733-42.

17. Chen W, Ma Y, Zhang H, et al. Reduning plus ribavirin display synergistic activity against severe pneumonia induced by $\mathrm{H} 1 \mathrm{~N} 1$ influenza A virus in mice. J Tradit Chin Med 2020;40:803-11.

18. Diagnosis and Treatment Program for Novel Coronavirus Infection Pneumonia (Trial Fifth Edition). Beijing: National Health Commission of the People's Republic of China and National Administration of Traditional Chinese Medicine of the People's Republic of China, 2020. Available online: http://www.gov.cn/zhengce/ zhengceku/2020-02/05/content_5474791.htm

19. Liu JL, Xu F, Zhou H, et al. Expanded CURB-65: a new score system predicts severity of community-acquired pneumonia with superior efficiency. Sci Rep 2016;6:22911. Erratum in: Sci Rep 2018;8:47005.

20. Jin YH, Cai L, Cheng ZS, et al. A rapid advice guideline for the diagnosis and treatment of 2019 novel coronavirus (2019-nCoV) infected pneumonia (standard version). Mil Med Res 2020;7:4.

21. Sharma B, Shahanshah MFH, Gupta S, et al. Recent advances in the diagnosis of COVID-19: a bird's eye view. Expert Rev Mol Diagn 2021. [Epub ahead of print]. doi: 10.1080/14737159.2021.1874354.

22. Chen N, Zhou M, Dong X, et al. Epidemiological and clinical characteristics of 99 cases of 2019 novel coronavirus pneumonia in Wuhan, China: a descriptive study. Lancet 2020;395:507-13.

23. Habibzadeh P, Stoneman EK. The novel coronavirus: a bird's eye view. Int J Occup Environ Med 2020;11:65-71.

24. Li G, De Clercq E. Therapeutic options for the 2019 novel coronavirus (2019-nCoV). Nat Rev Drug Discov 2020;19:149-50.

25. Arabi YM, Mandourah Y, Al-Hameed F, et al. Corticosteroid therapy for critically ill patients with middle east respiratory syndrome. Am J Respir Crit Care Med 2018;197:757-67.

26. Lansbury L, Rodrigo C, Leonardi-Bee J, et al. Corticosteroids as adjunctive therapy in the treatment of influenza. Cochrane Database Syst Rev 2019;2:CD010406.

27. Stockman LJ, Bellamy R, Garner P. SARS: systematic review of treatment effects. Plos Med 2006;3:e343.

28. Yang Y, Islam MS, Wang J, et al. Traditional Chinese medicine in the treatment of patients infected with 2019- 
new coronavirus (SARS-CoV-2): a review and perspective. Int J Biol Sci 2020;16:1708-17.

29. Gu S, Yin N, Pei J, et al. Understanding molecular mechanisms of traditional Chinese medicine for the treatment of influenza viruses infection by computational approaches. Mol Biosyst 2013;9:2696-700.

30. Romero MR, Serrano MA, Vallejo M, et al. Antiviral effect of artemisinin from Artemisia annua against a model member of the Flaviviridae family, the bovine viral diarrhoea virus (BVDV). Planta Med 2006;72:1169-74.

31. Haq FU, Roman M, Ahmad K, et al. Artemisia annua: Trials are needed for COVID-19. Phytother Res 2020;34:2423-4.

32. Efferth T, Romero MR, Wolf DG, et al. The antiviral

Cite this article as: $\mathrm{Xu} X$, Zhang J, Zheng W, Yang Z, Zhao X, Wang C, Su H, Zhao L, Xue L, Hu F, Xu X, Wen M, Liao J, Zeng Z, Wang L, Zeng J, Guo Y, Li B, Liu Q. Efficacy and safety of Reduning injection in the treatment of COVID-19: a randomized, multicenter clinical study. Ann Palliat Med 2021;10(5):5146-5155. doi: 10.21037/apm-20-2121 activities of artemisinin and artesunate. Clin Infect Dis 2008;47:804-11.

33. Zhou Z, Li X, Liu J, et al. Honeysuckle-encoded atypical microRNA2911 directly targets influenza A viruses. Cell Res 2015;25:39-49.

34. Guo S, Bao L, Li C, et al. Antiviral activity of iridoid glycosides extracted from Fructus Gardeniae against influenza A virus by PACT-dependent suppression of viral RNA replication. Sci Rep 2020;10:1897.

35. Wang F, Li CY, Zheng YF, et al. Identification of the allergenic ingredients in Reduning injection by ultrafiltration and high-performance liquid chromatography. J Immunol Res 2016;2016:4895672. 


\section{Supplementary}

\section{Appendix 1}

\section{Case definitions}

Diagnosis and Treatment Program for Novel Coronavirus Infection Pneumonia (Trial Fifth Edition, China) (1).

\section{Case for reporting}

At least one of the epidemiological histories (14 days before onset of symptoms):

(I) Travel or stay in Wuhan and surrounding cities and regions;

(II) Contact with a person diagnosed with COVID-19;

(III) Contact with a person from Wuhan and surrounding cities and regions;

(IV) Clustering occurrence.

Or at least two of the following clinical symptoms:

(I) Fever (measured or subjective) or respiratory symptoms;

(II) The total number of normal or decreased leukocytes;

(III) The total number of decreased lymphocytes.

\section{Clinical confirmed cases}

Suspected cases with radiographic evidence of pneumonia.

\section{Confirmed cases}

Clinical confirmed cases or suspected cases with at least one of following pathogenic evidence:

(I) Positive in SARS CoV-2 RT-PCR examination from respiratory tract or blood sample;

(II) High homology with SARS CoV-2 virus genomes in respiratory tract or blood sample sequencing.

\section{Clinical classification}

Two types of clinical classification were studied, including common and severe cases.

Common case:

(I) Fever (measured or subjective) or respiratory symptoms;

(II) Radiographic evidence of pneumonia

Severe case, at least one of the following symptoms:

(I) Respiratory distress, $\mathrm{RR} \geq 30$ times/min;

(II) Resting-state, mean oxygen saturation $\leq 93 \%$;

(III) $\mathrm{PaO}_{2} / \mathrm{FiO}_{2} \leq 300 \mathrm{mmHg}(1 \mathrm{mmHg}=0.133 \mathrm{kPa})$.

\section{Additional inclusion and exclusion criteria}

The full list of exclusion criteria, included:

(I) Diagnosed with COVID-19;

(II) One or more symptoms among fever, cough, and fatigue at enrollment;

(III) The time from onset to diagnosis time was not more than $48 \mathrm{~h}$; 
(IV) $\geq 18$ years of age.

The full list of exclusion criteria, included:

(I) Immunodeficiency, immunosuppressive drugs, or glucocorticoids within the past 3 months;

(II) Preparing for pregnancy, pregnant women, or lactating women;

(III) Allergies to more than two types of drugs or foods or allergies to ingredients of the trial drug;

(IV) Psychosis or those without self-knowledge ability;

(V) Estimated survival time less than $48 \mathrm{~h}$ from the start of screening;

(VI) Tracheal intubation was already done before screening;

(VII) Those who were judged by the investigators to be not suitable to participate in the trial.

The study was approved by the Ethics Committee of Tianjin University of Traditional Chinese Medicine. The study followed the GCP guidelines and the Declaration of Helsinki. Written informed consent was obtained from the patients or legal representatives. The study was registered (ChiCTR2000029589).

\section{Amendments}

We had an amendment on June 28, 2020. Secondary indicators including length of hospital stay and laboratory tests (leukocyte, lymphocyte count, neutrophil count, C-reactive protein, erythrocyte sedimentation rate, D-dimer improvement) were added to this trail research.
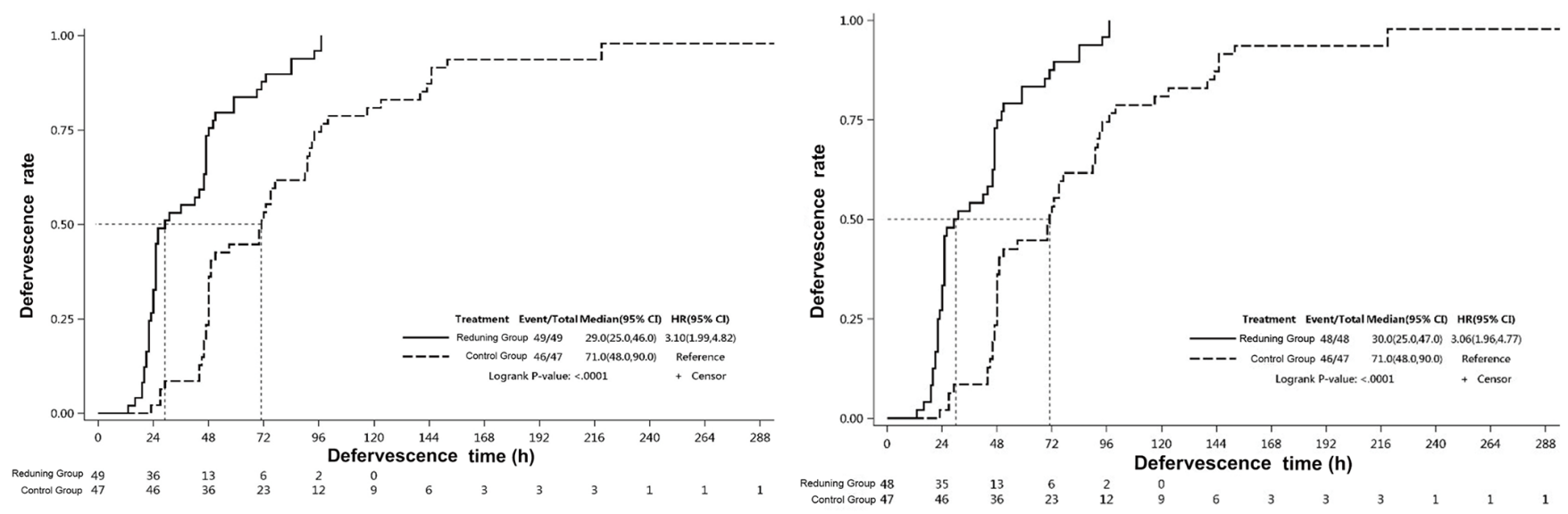

Figure S1 Time for defervescence. (A) Full-analysis set (FAS). (B) Per-protocol set (PPS). The blue line represents the control group.

Table S1 Survival rate at 28 days

\begin{tabular}{|c|c|c|c|c|c|c|}
\hline Time & \multicolumn{3}{|c|}{ FAS } & \multicolumn{3}{|c|}{ PPS } \\
\hline $28^{\text {th }}$ day, $\mathrm{n}(\%)$ & 77 (100.0) & 77 (96.3) & 0.2455 & $72(100.0)$ & 76 (96.2) & 0.2466 \\
\hline
\end{tabular}

FAS, full-analysis set; PPS, per-protocol set. 